
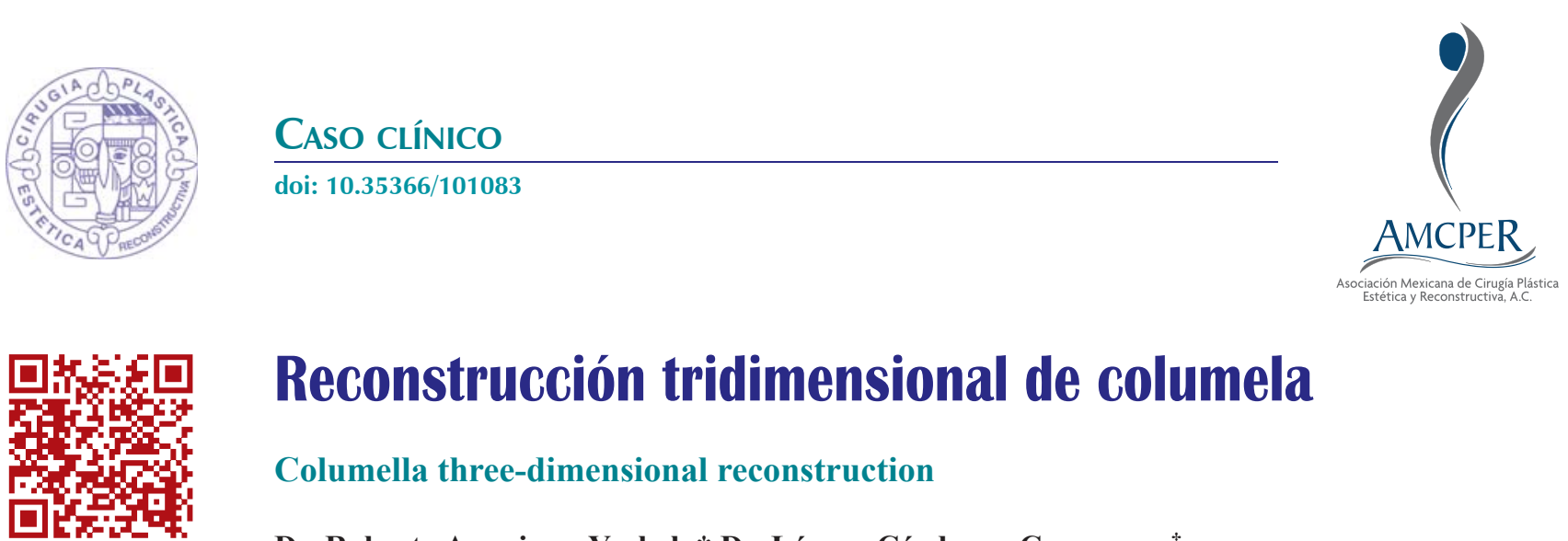

\title{
Reconstrucción tridimensional de columela
}

\author{
Columella three-dimensional reconstruction \\ Dr. Roberto Anguiano-Yazbek,* Dr. Lázaro Cárdenas-Camarena,* \\ Dr. Juan Aguilera-Serrano§
}

Palabras clave: Columela, nariz, reconstrucción, reconstrucción tridimensional.

Keywords: Columella, nose, reconstruction, three-dimensional reconstruction.

* Cirujano plástico y reconstructivo. Práctica privada Hospital Santa María Chapalita. Guadalajara, Jalisco. \$ Médico adscrito al Servicio de Cirugía Plástica del Instituto Jalisciense de Cirugía Reconstructiva« «Dr. José Guerrerosantos»» $\S$ Médico adscrito al Servicio de Cirugía Plástica del Hospital Centro Médico Nacional de Occidente IMSS.

Recibido:

27 octubre 2020

Aceptado:

15 febrero 2021

\begin{abstract}
RESUMEN
La pérdida de la columela puede ser causada por trauma, infecciones o lesiones malignas. Una reconstrucción óptima requiere un soporte estructural y una cobertura con textura y color y adecuados, aunado a que en el sito donador no quede un defecto o deformidad secundaria. Presentamos una técnica de reconstrucción tridimensional de columela en una paciente de tres años que nació a las 26 semanas de gestación, como consecuencia de lesión traumática causada por el uso del sistema de ventilación no invasivo en el manejo de la prematurez extrema, que provocó necrosis de la columela como complicación por la mascarilla. Diseñamos un colgajo trasversal en la parte superior del labio, dejando la parte central como pedículo cutáneo. Las alas del colgajo reconstruyen la cara lateral de la columela y el soporte se consigue con un poste de cartílago, logrando la reconstrucción tridimensional. La reconstrucción tridimensional se obtiene en un solo tiempo con un adecuado resultado estético y funcional, dando soporte firme a la punta nasal con el mismo color y textura. El defecto en el área donadora es casi nulo, mejorando funcionalmente la ventilación y el olfato. Concluimos que esta técnica permite la reconstrucción de la columela en un solo tiempo quirúrgico con un resultado estético y funcional adecuado, evitando deformidades en el sitio donador.
\end{abstract}

\section{ABSTRACT}

Columella loss can be caused by trauma, infection, or malignant injury. Optimal reconstruction requires structural support and coverage with adequate skin texture and color match, coupled with the absence of a secondary defect or deformity at the donor site. We present a three-dimensional columella reconstruction technique in a three-year-old patient, born at 26 weeks of gestation, as a consequence of a traumatic injury caused by the use of the non-invasive ventilation system in the management of extreme prematurity that caused necrosis of the columella as a complication from the use of a mask. We designed a transverse flap in the upper part of the lip, leaving the central part as a cutaneous pedicle. The flap wings reconstruct the lateral face of the columella and support is achieved with a cartilage graft, accomplishing three-dimensional reconstruction. The three-dimensional reconstruction is obtained in a single time with an adequate aesthetic and functional result, giving firm support to the nasal tip with the same color and texture. The defect in the donor area is almost nil, which improves ventilation and smell functionally. We conclude that this technique allows the reconstruction of the columella in a single surgical time with an adequate aesthetic and functional result, avoiding deformities in the donor site.

\section{INTRODUCCIÓN}

T a pérdida de la columela puede ser causada upor trauma, infecciones o lesiones malignas, con resultados devastadores tanto estéticos como funcionales. ${ }^{1}$ La ausencia congénita de la columela es rara, sólo hay unos cuantos casos reportados. $^{2}$

La subunidad de la nariz más difícil de reconstruir es la columela, debido a la limita-

ción de tejidos a su alrededor. ${ }^{3}$ Conseguir un resultado estético y funcional óptimo requiere de piel con el color y la textura adecuada y un soporte estructural subyacente, aunado a que el sito donador no cree un defecto o deformidad secundaria. 4

Se han reportado diferentes técnicas para la reconstrucción como colgajos del labio superior, ${ }^{5,6}$ marginales de las alas, ${ }^{7}$ con mucosa vestibular interna, ${ }^{8,9}$ nasomalar, ${ }^{10}$ reconstrucciones

Citar como: Anguiano-Yazbek R, Cárdenas-Camarena L, Aguilera-Serrano J. Reconstrucción tridimensional de columela. Cir Plast. 2021; 31 (1): 25-30. https://dx.doi.org/10.35366/101083 

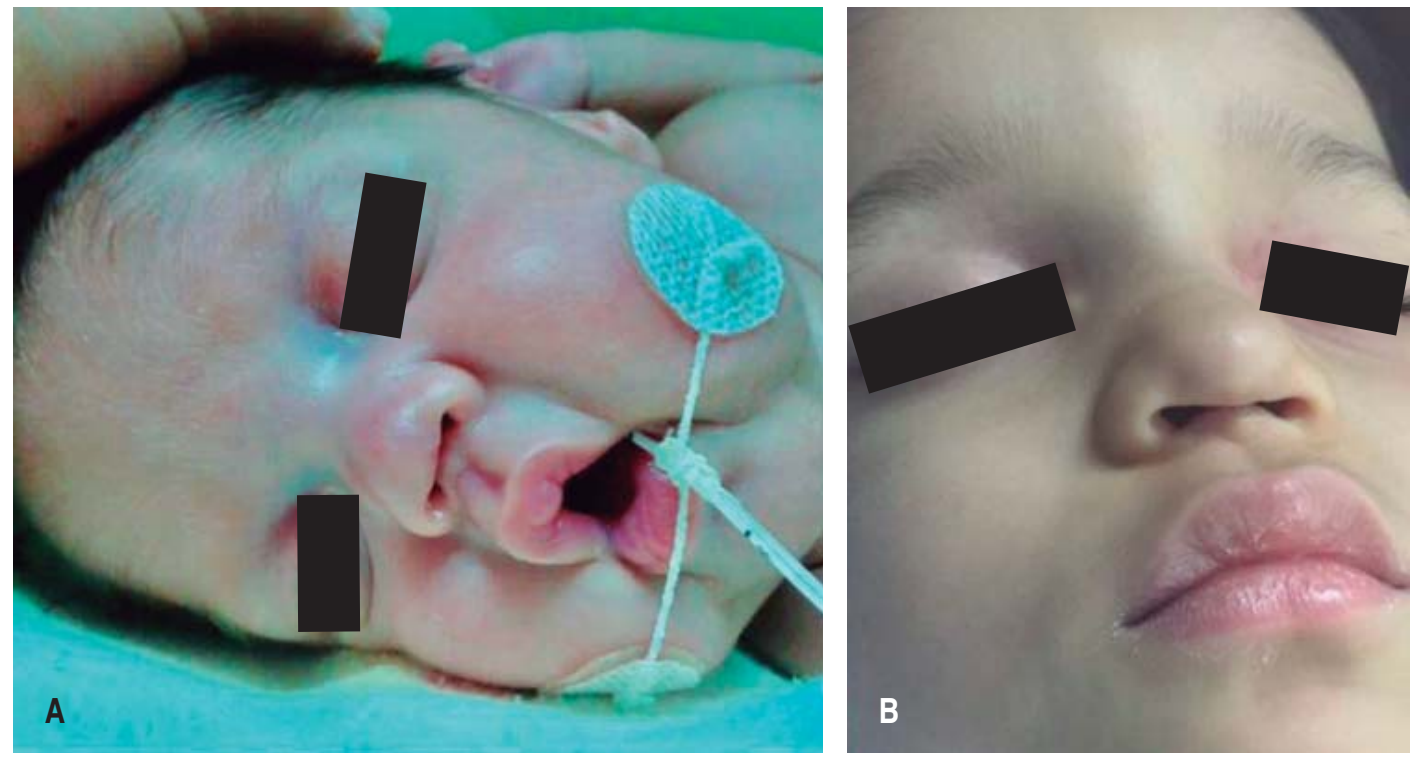

Figura 1: A) Recién nacida. B) Tres años de edad. Paciente con ausencia del tejido columelar, con colapso de la punta nasal, la cual toca prácticamente el nasión.

en un solo tiempo con tejidos distantes, injertos compuestos y colgajos libres vascularizados. ${ }^{11,12}$ Con todo y dicha multiplicidad de opciones, ninguna técnica parece cumplir con los requisitos funcionales y estéticos mencionados.

Nuestro objetivo es presentar una técnica de reconstrucción tridimensional de columela en un caso clínico pediátrico de tres años, debido a lesión traumática causada por el uso del sistema de ventilación no invasivo (SIPAP), como manejo de la prematurez extrema.

Presentamos un caso donde se realizó la reconstrucción con una modificación de la técnica descrita por Gillies ${ }^{5}$ y Earley, ${ }^{6}$ con un colgajo transverso del labio superior en la base de la nariz. La modificación consiste en elevar la base del colgajo y girar cada ala del mismo hacia la mucosa septal, así la base del colgajo reconstruye la pared anterior de la columela y las alas reconstruyen las paredes laterales al unirlas a la mucosa del septum, permitiendo la colocación de un injerto de cartílago para dar soporte y lograr una reconstrucción funcional tridimensional. Otra modificación a la técnica es el tomar un injerto de concha auricular, reduciendo así a un solo tiempo quirúrgico, ya que la técnica original se realiza en dos tiempos. ${ }^{5}$

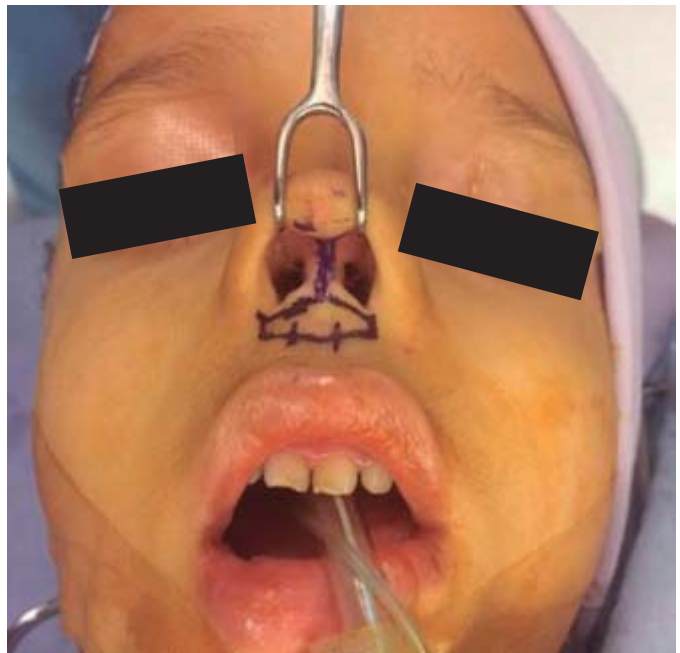

Figura 2: Se observa el septum caudal con mucosa nasal unida en la línea media. Diseñamos un colgajo trasversal en la parte superior del labio, cada ala del colgajo es de la longitud de la fosa nasal, con un ancho de $3 \mathrm{~mm}$.

\section{CASO CLÍNICO}

Paciente del sexo femenino de tres años de edad, que nació a las 26 semanas de gestación, presentando problemas funcionales respiratorios secundarios a la inmadurez pulmonar. La mas- 


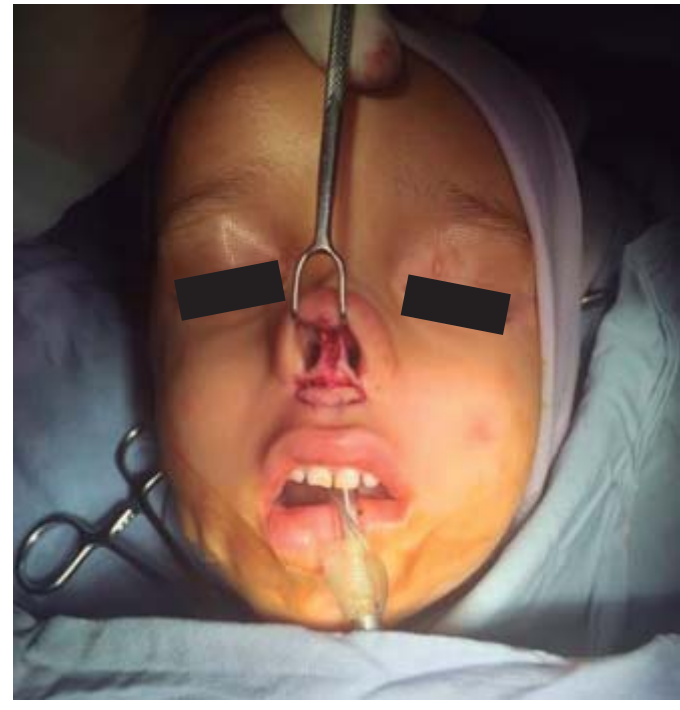

Figura 3: Se procede a realizar el corte del borde del colgajo, dejando la parte central inferior como pedículo cutáneo, se incide la mucosa septal en el borde caudal para disecarla en forma de libro abierto, realizamos la disección de la mucosa septal en ambos lados de 2 a 3 mm de longitud.

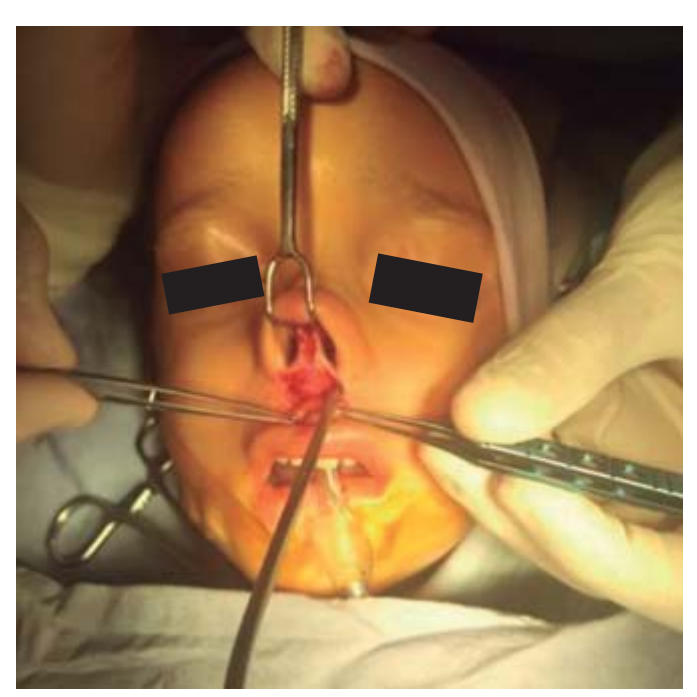

Figura 4: Rotamos las alas del colgajo para unir la parte posterior a la mucosa septal. 10010101011

carilla ocasionó necrosis de la columela como complicación, ya que la paciente estuvo en la Unidad de Cuidados Intensivos Neonatales (UCIN), donde se manejó con el sistema de ventilación no invasivo (SIPAP) por varias semanas.
A la exploración se encontró ausencia del tejido columelar, con colapso de la punta nasal, la cual toca prácticamente la parte superior del filtrum. A la rinoscopia se observa el septum caudal con mucosa nasal unida en la línea media y disminución en el sentido del olfato (Figuras 1 y 2).

\section{Técnica quirúrgica}

Diseñamos un colgajo transversal en la parte superior del labio, cada ala del colgajo es de la longitud de la fosa nasal, con un ancho de $3 \mathrm{~mm}$ (Figura 2). Incidimos la mucosa septal en el borde caudal para disecarla en forma de libro abierto, realizamos la disección de la mucosa septal en ambos lados de 2 a 3 $\mathrm{mm}$ de longitud para poder realizar el cierre. Se procede a efectuar el corte del borde del colgajo dejando la parte central inferior como pedículo cutáneo (Figura 3). Disecamos de la periferia al centro del colgajo en ambas alas en el plano superior del músculo orbicular,
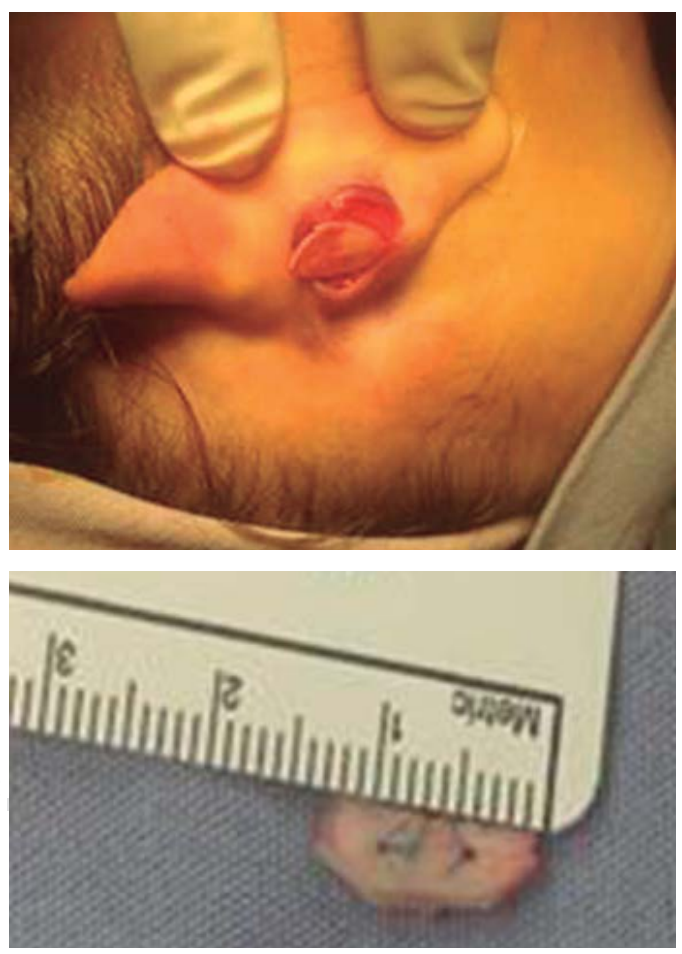

Figura 5: Realizamos la toma de un injerto de cartílago de concha auricular en forma oval, el cual se debilita en la cara convexa y se unen dos fragmentos con polipropileno. 


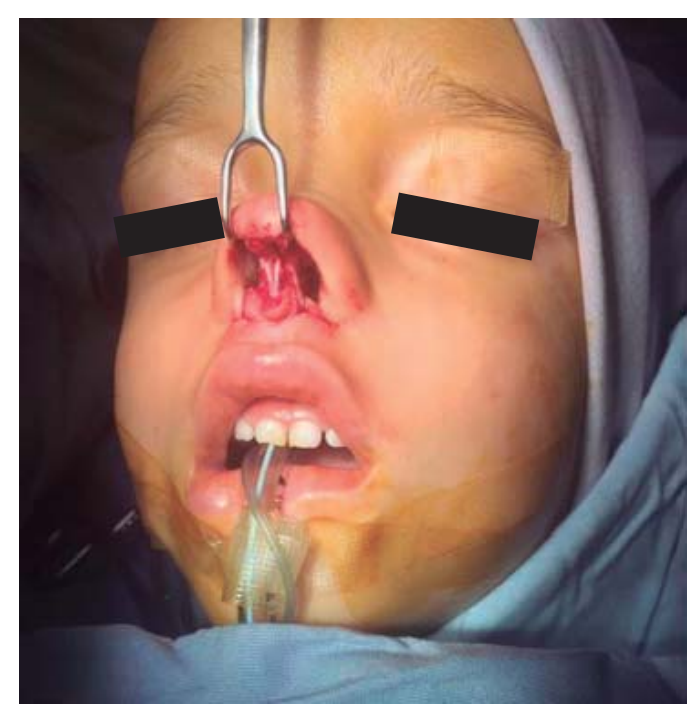

Figura 6: Colocamos en el bolsillo de la base del colgajo el poste de cartílago, para dar soporte a la punta nasal.

dejando un colgajo cutáneo de pedículo central (Figura 4). Tomamos un injerto de cartílago de concha auricular en forma oval, el cual se debilita en la cara convexa para unir los dos fragmentos con polipropileno, logrando mayor soporte del que será el poste de cartílago (Figura 5). Se avanza la base del colgajo para afrontar los bordes del lecho dejado al levantar el colgajo. Se coloca el poste de cartílago en el bolsillo de la base del colgajo para darle soporte a la punta nasal, se procede a rotar las alas del colgajo para unir la parte posterior a la mucosa septal (Figura 6); la parte superior se une a la piel de la punta nasal, la región anterior de las alas del colgajo se une entre sí para formar la parte caudal de la columela. De esta manera, se deja el poste de cartílago cubierto y se realiza la reconstrucción tridimensional (Figuras 7 y 8). Las suturas se realizan con monocryl 5-0.

En el postoperatorio inmediato obtuvimos una proyección satisfactoria de la punta nasal, con una reconstrucción completa de la columela en un solo tiempo quirúrgico, consiguiendo un soporte adecuado.

A los cinco meses se conservó la proyección y sostén de la nariz. Por otro lado, como reacción al material de sutura se presentó un pequeño granuloma, mismo que se programó para su resección, aunque los padres prefirieron esperar a que la paciente tuviera mayor edad. Continuamos el seguimiento por un periodo de cuatro años y medio, a esta edad apreciamos una buena función ventilatoria y en el aspecto estético se observa que se conserva una forma adecuada de la columela con proyección de la punta nasal, una cicatriz prácticamente imperceptible con coloración similar al resto de la nariz (Figuras 9 a 12).

\section{DISCUSIÓN}

Se han reportado diferentes técnicas para la reconstrucción de la columela en uno o varios tiempos quirúrgicos. ${ }^{5-12}$

Para lograr una reconstrucción adecuada con un resultado estético y funcional, se requiere de piel del mismo color y textura, con un soporte adecuado que no deje un defecto secundario en el sitio donador. Algunas técnicas

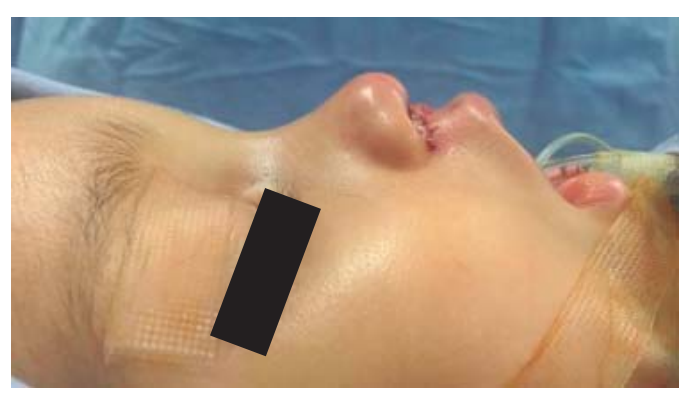

Figura 7: Vista de perfil con proyección adecuada de la punta.

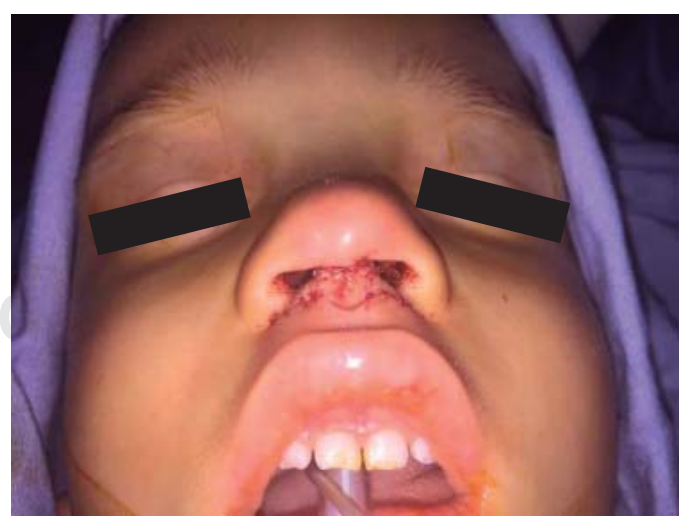

Figura 8: Vista caudal, la piel es del mismo color con una cicatriz mínima. 


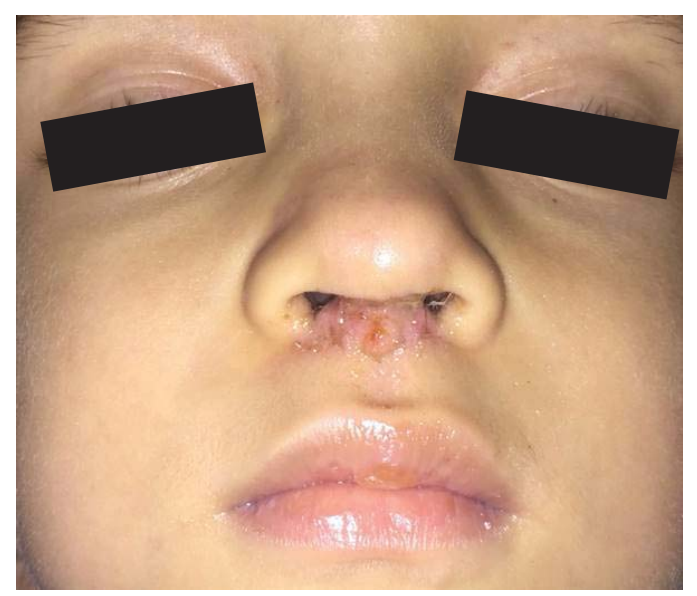

Figura 9: Un mes postoperada, cicatriz mínima.

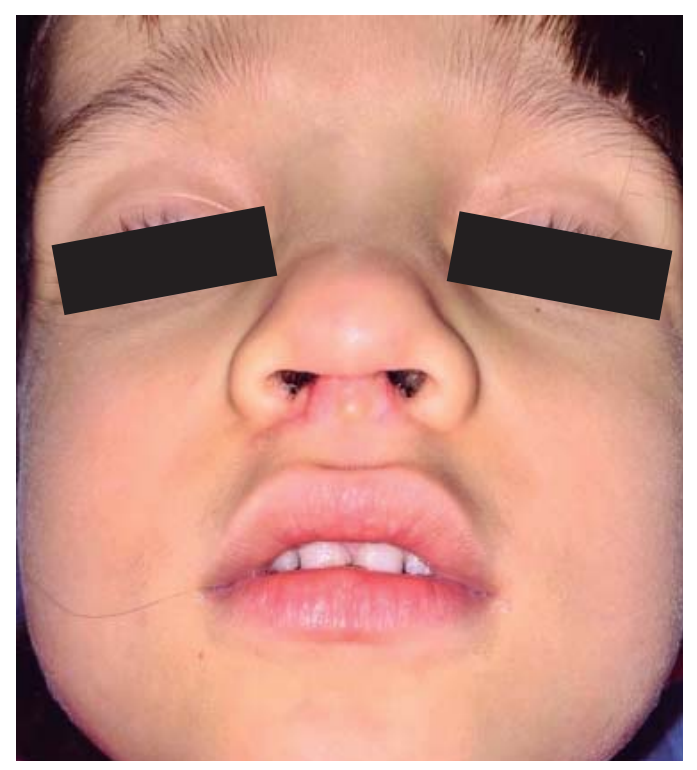

Figura 10: Dos meses postoperada, se observa un granuloma en la base de la columela, menor inflamación, buen soporte y proyección de la punta nasal.

descritas, como los colgajos libres ${ }^{12}$ o los colgajos frontales, ${ }^{7}$ no cumplen estos principios, dejando secuelas estructurares y diferencias en el color. Con esta técnica logramos una reconstrucción tridimensional en un solo tiempo quirúrgico, con un adecuado resultado estético y funcional, dando soporte firme a la punta nasal con el mismo color y textura. El defecto en el área donadora es casi nulo y la cicatriz es casi imperceptible. Funcionalmente mejora la ventilación, con una mejoría en el sentido del olfato.

\section{CONCLUSIÓN}

Esta técnica permite la reconstrucción de la columela en un solo tiempo quirúrgico. La textura y color son adecuados, ya que es piel de la misma zona. Logramos un soporte adecuado de la punta nasal, evitando el colapso. Se evitan deformidades en el sitio donador con cicatriz casi nula y un cierre adecuado, sin tensión o retracciones. Es un procedimiento más sencillo de realizar que un colgajo a distancia o libre. La ventilación mejora y, por consiguiente, también el sentido del olfato. El resultado es permanente al pasar de los años.

\section{AGRADECIMIENTOS}

A la Dra. Liliana Castillejos-Zenteno por ayudarnos con la corrección de estilo, Dr. Jorge Alcaraz López por ayudarnos a tomar las fotografías.

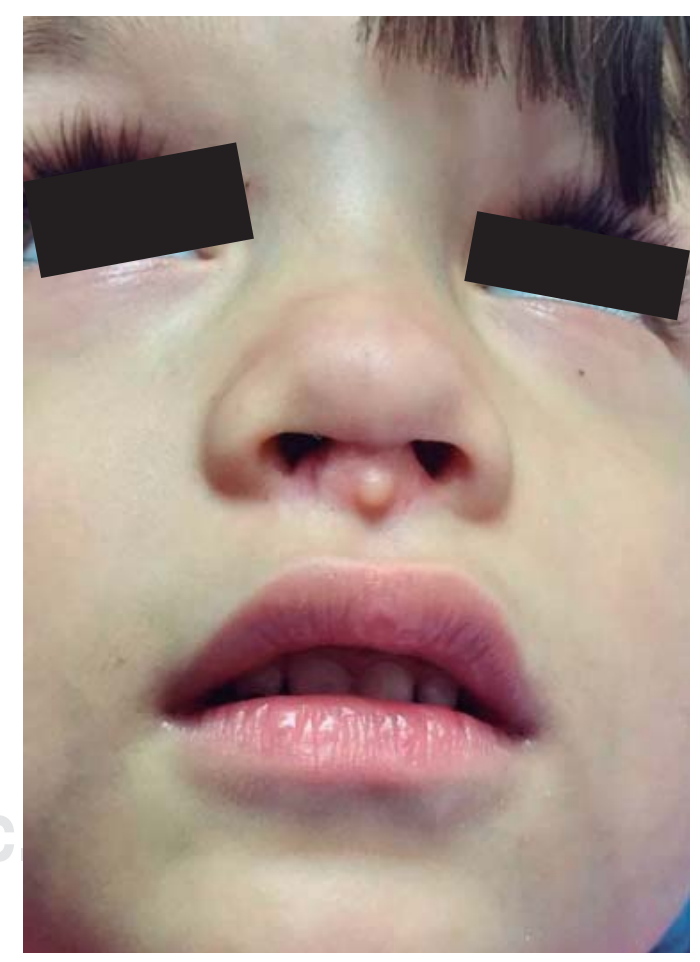

Figura 11: Seis meses postoperada, la coloración es muy parecida al resto de la nariz, cicatriz casi imperceptible, el granuloma como respuesta al material de sutura sin cambios. 


\section{Figura 12:}

A los cuatro años y medio de seguimiento la cicatriz es casi nula, buen soporte y proyección de la punta nasal, el color de la columela es igual que el resto de la nariz, el granuloma continua presente, se programará

para la resección.
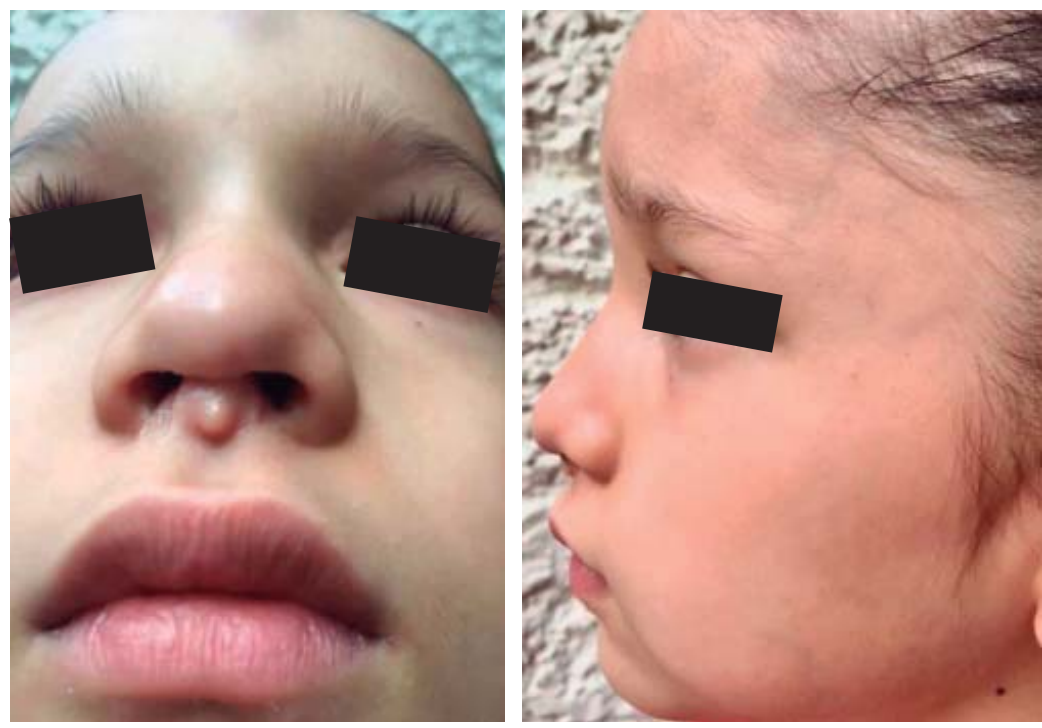

\section{REFERENCIAS}

1. Margulis A, Bauer BS, Han H, Patel PK. Reconstruction of the columella in a pediatric patient. Plast Reconstr Surg 2003; 112: 1948-1949.

2. Mavili ME, Akyurek M. Congenital isolated absence of the nasal columella: reconstruction with an internal nasal vestibular skin flap and bilateral labial mucosa flaps. Plast Reconstr Surg 2000; 106: 393-399.

3. Smith V, Papay FA. Surgical options in columellar reconstruction. Otolaryngol Head Neck Surg 1999; 120: 947 .

4. Morrison CM, Bond JS, Leonard AG. Nasal reconstruction using the Washio retroauricular temporal flap. Br J Plast Surg 2003; 56: 224-229.

5. Gillies H. The columella. Br J Plast Surg 1949; 2: 192-201.

6. Earley MJ, Chantarasak ND. The transverse forked flap in columella reconstruction. Br J Plast Surg 1989; 42: 270-273.

7. Saad MN, Barron JN. Reconstruction of the columella with alar margin flaps. Br J Plast Surg 1980; 33: 327-329.

8. Balandini D, Tremolada C, Beretta M, Mascetti M. latrogenic nostril stenosis: Aesthetic correction using a vestibular labial mucosa flap. Plast Reconstr Surg 1995; 95: 569-571.
9. Juri J, Juri C, Belmont JA et al. Neighboring flaps and cartilage grafts for correction of serious secondary nasal deformities. Plast Reconstr Surg 1985; 76: 876-881.

10. Kaplan I. Reconstruction of the columella. Br J Plast Surg 1972; 25: 37-38.

11. Paletta FX, VanNorman RT. Total reconstruction of the columella. Plast Reconstr Surg 1962; 30: 322-328.

12. Tanaka Y, Tajima S, Tsujiguchi K et al. Microvascular reconstruction of nose and ear defects using composite auricular free flaps. Ann Plast Surg 1993; 31: 298-302.

Conflicto de intereses: Los autores declaran no tener conflicto de intereses.

Correspondencia:

Dr. Roberto Anguiano-Yazbek

Calle Santa Rita Núm. 1031, $2^{\circ}$ piso, Consultorio 212,

Col. Jardín de San Ignacio Chapalita, 45040,

Zapopan, Jalisco, México.

E-mail: dranguiano@yahoo.com.mx 